\title{
Review
}

nephron

Clinical

Practice

Published online: September 24, 2014

\section{Statins for the Prevention of Contrast-Induced Acute Kidney Injury}

\author{
Timothy Ball Peter A. McCullough \\ Baylor University Medical Center, Baylor Heart and Vascular Institute, Baylor Jack and Jane Hamilton Heart and \\ Vascular Hospital, Dallas, Tex., and The Heart Hospital, Plano, Tex., USA
}

\section{Key Words}

Statins $\cdot$ Acute kidney injury $\cdot$ Contrast

\begin{abstract}
Acute kidney injury (AKI) is a common medical problem, especially in patients undergoing cardiovascular procedures. The risk of kidney damage has multiple determinants and is often related to or exacerbated by intravenous or intra-arterial iodinated contrast. Contrast-induced AKI (CI-AKI) has been associated with an increased risk of subsequent myocardial infarction, stroke, the development of heart failure, rehospitalization, progression of chronic kidney disease, end-stage renal disease, and death. Statins have been studied extensively in the setting of chronic kidney disease and they have been shown to reduce albuminuria, but they have had no effect on the progressive reduction of glomerular filtration or the need for renal replacement therapy. Several meta-analyses have shown a protective effect of short-term statin administration on $\mathrm{Cl}-\mathrm{AKI}$ and led to two large randomized controlled trials evaluating the role of rosuvastatin in the prevention of $\mathrm{Cl}-\mathrm{AKI}$ in high-risk patients with acute coronary syndrome and diabetes mellitus. Both trials showed a benefit of rosuvastatin prior to contrast ad-
\end{abstract}

ministration in a statin-naive patient population. In aggregate, these studies support the short-term use of statins specifically for the prevention of $\mathrm{Cl}-\mathrm{AKI}$ in patients undergoing coronary angiography with or without percutaneous coronary intervention.

(c) 2014 S. Karger AG, Base

\section{Introduction}

Acute kidney injury (AKI) is a common problem especially in the hospitalized patient. AKI has been on the rise over the past decade. A study from the Nationwide Inpatient Sample found that from 2000 to 2009 the incidence of dialysis-requiring AKI increased from 222 to 533 cases per million person-years, averaging a $10 \%$ increase per year. Additionally, the total number of deaths associated with dialysis-requiring AKI rose from 18,000 in 2000 to nearly 39,000 in 2009 [1].

Changing Paradigms in Acute Kidney Injury: From Mechanisms to Management - Proceedings of the 5th Annual UAB-UCSD O'Brien Center Symposium (San Diego, Calif., USA, March 4, 2014).

\section{KARGER}

E-Mail karger@karger.com

www.karger.com/nec
(C) 2014 S. Karger AG, Basel

$1660-2110 / 14 / 1274-0165 \$ 39.50 / 0$
Peter A. McCullough, MD, MPH

Baylor Heart and Vascular Institute 621 N. Hall St., H030

Dallas, TX 75226 (USA)

E-Mail peteramccullough@gmail.com 
Fig. 1. Putative mechanisms for the beneficial effect of statins in preventing CI-AKI.

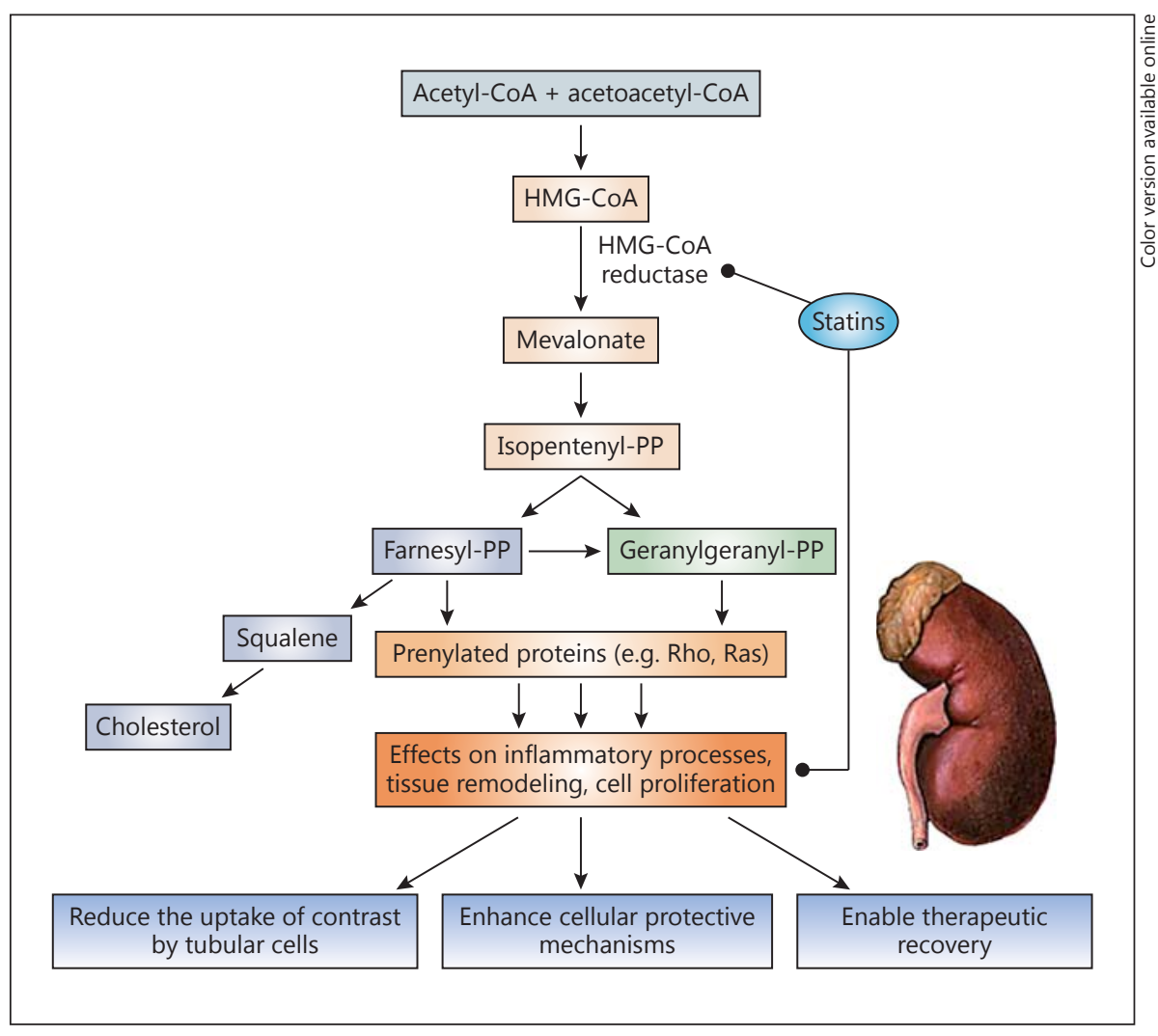

As the kidneys are susceptible to injury from multiple sources, the etiology of AKI can vary widely. Among hospitalized patients, the most common causes of AKI include acute tubular necrosis from ischemia, nephrotoxin exposure, and sepsis [2]. These insults can be a direct result of the patient's disease but are often an unfortunate adverse event related to diagnostic or therapeutic interventions. Specifically, cardiac surgery and percutaneous coronary intervention can be harmful by exposing the kidney to intraoperative hypotension, postoperative cardiac complications that impair renal perfusion, hemolysis, atheroemboli, and iodinated contrast media which probably causes some renal tubular epithelial damage in every case.

The incidence of postoperative AKI varies widely in the literature based on the definition of AKI used in the study. One recent, large series of 2006 from the Society of Thoracic Surgery found that the rate of AKI (defined as an increase of creatinine to $>2 \mathrm{mg} / \mathrm{dl}$ with a minimum doubling of the preoperative value, or a new requirement for dialysis) ranged from 3.6 to $5 \%$ [primarily coronary artery bypass graft (CABG) surgery] and the inci- dence increased to $7.5-12.9 \%$ when CABG was combined with an aortic or mitral valve. The incidence of contrast-induced AKI (CI-AKI) varies greatly in patients, i.e. from $<1$ to $10-20 \%$, depending on risk factors. Multiple risk calculators are available to predict CI-AKI and they generally include age, severity of heart failure, baseline renal function, and diabetes. Also, the risk of AKI is higher during interventional exposures compared to imaging due to the risk of atheroemboli (particularly during transcatheter aortic valve replacement). CI-AKI is usually temporary and most patients return to their baseline or near baseline glomerular filtration rate (GFR). Historically, CI-AKI specifically and AKI generally were thought to be transient phenomena, without lasting effects if a patient's GFR recovered to normal. More recent data suggest that any significant AKI may result in lasting damage. A meta-analysis of patients found AKI to be an independent risk factor for the development of chronic kidney disease (CKD), end-stage renal disease, cardiovascular events, heart failure, and increased mortality $(\mathrm{HR}=2.0)$ compared to patients without AKI [3]. The impact of AKI following surgery 


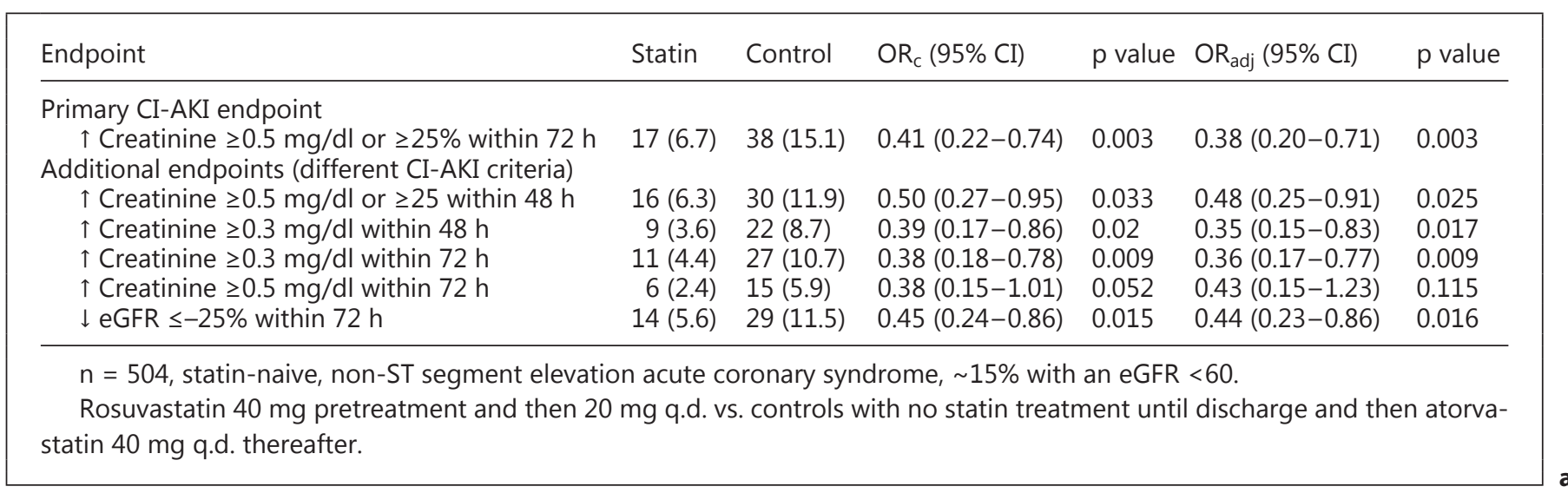

Fig. 2. Primary results of the PRATO-ACS trial. Adapted from Leoncini et al. [22]. a An unconditional logistic model was applied, and crude ORs with 95\% CI were reported. Adjusted ORs were controlled for sex, age, diabetes, hypertension, LDL cholesterol values, creatinine clearance at baseline, ejection fraction, contrast volume, and CI-AKI risk score. b Primary endpoint occurrence (an increase in serum creatinine concentration $\geq 0.5 \mathrm{mg} / \mathrm{dl}$ or $\geq 25 \%$ over baseline within $72 \mathrm{~h}$ ) in the 2 study groups. $\mathrm{OR}_{\mathrm{c}}=$ Crude $\mathrm{OR} ; \mathrm{OR}_{\mathrm{adj}}=$ adjusted OR.

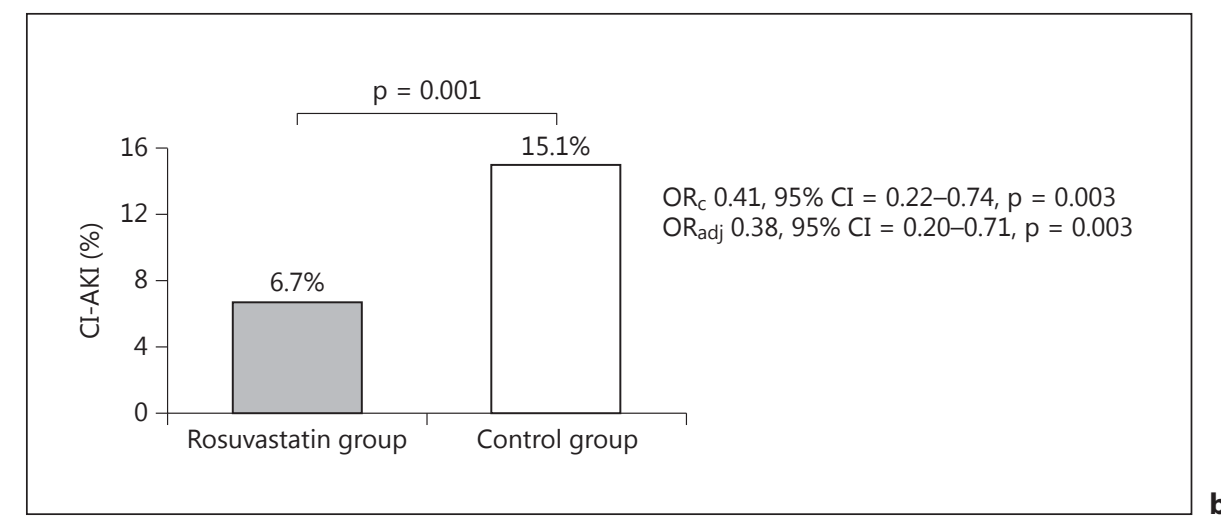

is even more severe. In a prospective cohort study of 43,642 patients undergoing CABG or valvular surgery, AKI following surgery resulted in a 30-day mortality rate of $64 \%$, compared to $4 \%$ in patients without renal failure [4]. A more recent study showed that survival has improved over time but remains significant, with a $23 \%$ in-hospital mortality rate for postoperative AKI and a $49 \%$ mortality rate for patients with AKI requiring dialysis [5]. In general, the more severe the injury with higher elevations in serum creatinine, oliguria, and more sustained acute injury over several days, the greater the chance of permanent renal damage, need for dialysis, or death.

\section{Statins and the Risk of Rhabdomyolysis and AKI}

Seven statins (lovastatin, simvastatin, pravastatin, fluvastatin, atorvastatin, rosuvastatin, and pitavastatin) are currently available in the USA. The pharmacodynamics of these medications varies widely but, with the exception of pravastatin, these medications are at least partly metabolized by the CYP450 system. The CYP3A family metabolizes lovastatin, simvastatin, and atorvastatin, whereas CYP2C9 metabolizes fluvastatin and rosuvastatin. This elimination can be greatly affected by inhibition of the CYP450 system. There is a long list of medications that can affect this system. Coadministered grapefruit juice has been shown to inhibit the CYP3A4 system in the gastrointestinal mucosa, thereby increasing bioavailability and the concentration of lovastatin, simvastatin, and atorvastatin. Cyclosporine is a prototypic example of a potent CYP3A4 and P-glycoprotein inhibitor. Inhibition of the CPY450 system with such medications can cause a significant elevation of statin blood levels, leading to an increased risk of myalgias, myopathy, and rhabdomyolysis. Initially, it was thought that creating hydrophilic statins would help prevent muscle damage by preventing diffusion across the muscle cell membrane, but this is not the case. It has now been shown that a set of influx and efflux transporters play a key role in regulating the level of statins in the skeletal muscle. Interestingly, rosuvasta- 


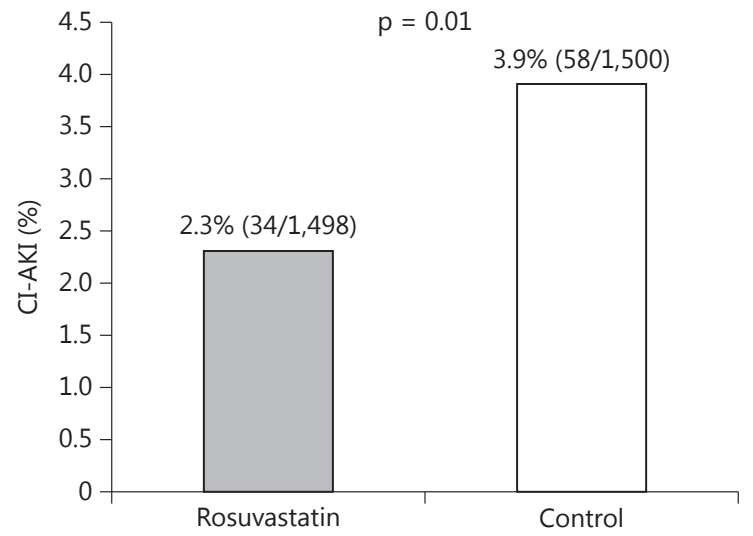

- $\mathrm{n}=2,998$, stable CKD, eGFR 30-60, undergoing angiography

- $58 \%$ statin-naive

- $42 \%$ statins washed out

- Rosuvastatin $10 \mathrm{mg}$ q.d. for 2 days prior to and 3 days after the procedure vs. no statin for 5 days and then return to usual care

Fig. 3. Primary results from the trial by Han et al. [23] in elective angiography. Adapted from Han et al. [23].

tin and atorvastatin are substrates of some of the efflux transports, which may explain why increasing doses of these medications do not lead to a high incidence of myopathy [6].

All statins and some of their early metabolic breakdown products (statin alcohols) are directly toxic to skeletal myocytes, particularly at high maximal blood concentrations. Statins have been associated with skeletal muscle damage via several mechanisms including negative membrane interactions, inhibition of cholesterol synthesis, a decreased concentration of intermediate proteins, accumulation of intracellular lipids and sterols, and a decreased number of mitochondria within skeletal myocytes [7]. Rhabdomyolysis is a rare but feared complication of this damage. A large 252,460 patient study found the incidence in patients on statin monotherapy (atorvastatin, pravastatin, or simvastatin) to be 0.44 per 10,000 person-years [8]. The incidence has been shown to increase when the medication is given in combination with cyclosporine or gemfibrozil. Acute rhabdomyolysis can lead to electrolyte disturbances and heart arrhythmias; however, the most significant complication associated with the disease is AKI. Renal tubular damage is caused by the release of myoglobin into the circulation, leading to mechanical obstruction of renal tubules and the direct toxic effect of free chelatable iron, which catalyzes oxidative stress reactions within renal tubular cells and causes cell-to-cell death. The incidence of AKI in rhabdomyolysis varies from approximately 15 to $50 \%$ depending on the cohort examined [9], thus carrying a significantly worse prognosis. One study examining rhabdomyolysis in an ICU population found the mortality rate to be $59 \%$ with AKI compared to $22 \%$ without [10]. Although the incidence is very rare, it has generated a large amount of discussion in the literature.

Likely due to the exaggerated concern for statin therapy leading to AKI, rosuvastatin in 80-mg doses drew controversy when it was shown to increase proteinuria. Further studies found that the increase was due to tubular secretion of low-molecular-weight (nonalbumin) proteins which was neither associated with nor promoted glomerular toxicity or tubular toxicity [11]. The FDA did approve the medication but only at doses ranging from 5 to $40 \mathrm{mg}$.

\section{Meta-Analyses of Small Statin Trials for Prophylaxis of CI-AKI}

Statins may be renoprotective via several mechanisms. Broadly, they help through inhibition of cholesterol synthesis as reabsorption of lipoproteins by the kidney tubules accelerates inflammation, and deposition in the glomerular mesangium promotes glomerular sclerosis. Also, statins have beneficial pleiotropic effects including attenuation of endothelial dysfunction and oxidative stress, anti-inflammation, antiproliferation of mesangial cells, and protection of podocytes [12]. A number of recent meta-analyses have examined the possible benefit of statins in CKD as well as AKI, specifically CI-AKI.

In 2006, Sandhu et al. [13] performed a meta-analysis including 27 studies with 39,704 participants that found a statistically significant slower decline in eGFR in patients on statins; this benefit was found to be most significant in patients with cardiovascular disease. Patients on statins were also found to have a significant improvement in proteinuria [13]. Also in 2006, Douglas et al. [14] published a meta-analysis analyzing proteinuria; it included 15 studies and 1,384 patients and concluded that statins may reduce proteinuria, with the greatest impact on patients with pathologic levels of proteinuria [14]. Another meta-analysis in 2008 by Strippoli et al. [15] looked at statin use in patients with $\mathrm{CKD}$; they examined 50 trials 
assessing various endpoints including proteinuria, renal function, and mortality. They included 6 trials looking at proteinuria and found a change of $-0.73 \mathrm{~g} / 24 \mathrm{~h}$. They did not find a significant improvement in GFR or mortality [15]. In 2009, Navaneethan et al. [16] wrote a Cochrane Review analyzing the effects of statins in patients with CKD (not on dialysis). They included 26 studies with 25,017 participants and found a significant benefit in allcause mortality and cardiovascular mortality. They also found a significant decrease in proteinuria but no significant benefit in renal function based upon serum creatinine. Interestingly, they found no increase in rhabdomyolysis or elevated liver enzymes in patients taking statins compared to a placebo [16]. In 2012, Wu et al. [12] performed a meta-analysis with 16 trials and 24,278 participants analyzing the possible renal benefit of rosuvastatin and atorvastatin and found a significant improvement in proteinuria in the atorvastatin group and a significant improvement in GFR in the atorvastatin-and-rosuvastatin group [12].

In addition to the growing evidence of statin therapy in $\mathrm{CKD}$, there is evidence that statins may have a benefit in CI-AKI. In 2011, Pappy et al. [17] performed a metaanalysis looking at the benefit of statin therapy in CIAKI, including 10 trials ( 3 randomized controlled trials and 7 nonrandomized studies). They did not show a significant benefit in the randomized trials but they did show a marginal benefit in the incidence of CI-AKI in the nonrandomized studies [17]. Also in 2011, Zhang et al. [18] performed a meta-analysis including 4 trials and 751 patients and found no significant improvement in CIAKI in patients pretreated with a statin compared to nonstatin controls, but they did note a slight improvement in serum creatinine [18]. In 2011, Zhang et al. [19] looked at the benefit of a high-dose statin for the prevention of CI-AKI, and in a meta-analysis including 8 studies and 1,423 patients they found a significant improvement in serum creatinine and the incidence of CI-AKI [19]. Similarly, Zhou et al. [20], in a meta-analysis in 2011 including 5 trials and 1,009 patients, found that shortterm, high-dose statins were beneficial in reducing the incidence of CI-AKI. On subgroup analysis, this benefit was only shown to be significant in patients with CKD stage 3 or higher [20]. In 2012, Li et al. [21] performed a meta-analysis that again looked at the benefit of shortterm high-dose statins in the prevention of CI-AKI; 7 studies and 1,399 patients were included. They found a significant improvement in the incidence of CI-AKI but no benefit in AKI requiring dialysis [21]. As with all meta-analyses, these studies are limited by multiple con- founding factors, especially reporting bias; however, these observations have led to larger randomized controlled trials.

\section{Larger Randomized Trials}

Recent larger, high-quality randomized controlled trials seem to confirm the renal benefit of statins. The Protective Effect of Rosuvastatin and Antiplatelet Therapy on Contrast-Induced Acute Kidney Injury and Myocardial Damage in Patients with Acute Coronary Syndrome (PRATO-ACS) trial analyzed the role of rosuvastatin in statin-naive patients for preventing CI-AKI. They randomized statin-naive non-ST elevation acute coronary syndrome patients undergoing percutaneous coronary intervention to statin or no-statin therapy. Statin patients received $40 \mathrm{mg}$ rosuvastatin on admission and then $20 \mathrm{mg}$ daily, while control patients did not receive statins during the hospitalization. At discharge, the statin group continued treatment with $20 \mathrm{mg} /$ day rosuvastatin $(10 \mathrm{mg} /$ day for patients with a GFR $<30 \mathrm{ml} / \mathrm{min} / \mathrm{m}^{2}$ ), while controls received $40 \mathrm{mg} /$ day atorvastatin. CI-AKI was defined as a rise in creatinine of $0.5 \mathrm{ml} / \mathrm{dl}$ or $25 \%$ above baseline within $72 \mathrm{~h}$ of receiving contrast. The study found that the statin group had a significantly lower rate of CI-AKI than the no-statin group (6.7 vs. $15.1 \%$; adjusted OR 0.38; $95 \%$ CI $0.20-0.71 ; \mathrm{p}=0.003)$. This benefit was significant across all prespecified risk categories. In addition to the primary endpoint, multiple positive secondary endpoints were noted. There was decrease in 30-day composite death, dialysis, myocardial infarction, stroke, or persistent renal damage in the statin group (3.6 vs. $7.9 \%$, respectively; $\mathrm{p}=0.036$ ), as well as a trend towards a decrease in death or myocardial infarction at 6 months (3.6 vs. 7.2\%, respectively; $\mathrm{p}=0.07$ ) [22].

Han et al. [23] tested rosuvastatin for the prevention of CI-AKI in patients with diabetes and CKD who were undergoing coronary or peripheral angiography with or without intervention. Patients who were on statins previously were asked to hold their statins for 14 days prior to the procedure. Patients were randomized to statin therapy or the standard of care (no statin). The statin group received $10 \mathrm{mg}$ rosuvastatin daily starting 2 days before the procedure and continuing for 5 days. The standardof-care group and the statin group resumed statin therapy 3 days after intervention. CI-AKI was defined as an increase in serum creatinine concentration $\geq 0.5 \mathrm{mg} / \mathrm{dl}$ or $0.25 \%$ above baseline at $72 \mathrm{~h}$. The statin group had a significantly lower incidence of CI-AKI (2.3 vs. $3.9 \%$; $p=$ 
0.01 ). Additionally, a significantly lower rate of heart failure was noted in the statin group at the 30-day follow-up (2.6 vs. $4.3 \%$; $\mathrm{p}=0.02)$ [23].

As studies demonstrating the possible renoprotective effects of statins accumulate, the evidence is becoming harder to ignore. Until this year, the studies were limited in quality and were generally small cohort studies compiling into meta-analyses. While informative, they suffer from several inherent flaws. However, with the addition of these two large well-designed randomized controlled trials there can be little question that there is some renal benefit of statins in high-risk populations. The exact mechanism of this protective effect has been postulated; however, the full story does not seem to have been written. The benefits of cholesterol inhibition and the pleiotropic effects of statins have been discussed previously. Additionally, statins may show a benefit specifically in CI-AKI. Interestingly, in the rosuvastatin trial high doses were shown to cause an elevation in urine protein. As discussed above, these proteins were found to be low-molecular-weight proteins. Initially, there was some concern that this increase in proteinuria was related to glomerular damage, but further studies suggest a more benign etiology. The effects of statin inhibition of HMG-CoA reductase are not limited to lower LDL. Statins lower isoprenoid pyrophosphates which play a key role in the proper function of GTP-binding proteins [24]. GTP-binding proteins are responsible for multiple cellular functions including endocytosis. In an in vitro model, rosuvastatin inhibited endocytosis in cells found in the renal tubules [25]. Decreased reabsorption leads to an increase in pro- teinuria and also provides a possible mechanism for the benefit in CI-AKI. Contrast is known to cause direct toxicity to renal cells secondary to the iodine present in the media. Also, contrast is known to persist in the kidneys for several days following contrasted studies. The mechanism of this is not clear, but it is possible that a portion of the excreted contrast is reabsorbed in the renal tubule and then persists in the peritubular space. If statins prevent the reabsorption of protein in the tubule, it is possible that the reabsorption of contrast is also prevented, thereby decreasing the kidney exposure to contrast and helping to prevent CI-AKI.

\section{Conclusions}

Serious skeletal muscle toxicity is rare with statins, and one meta-analysis suggested that this complication was not more common in CKD than in the general population [16]. Rhabdomyolysis, when it occurs, is associated with high rates of AKI. At high doses, rosuvastatin was associated with nonalbuminuric proteinuria and hence the 80 $\mathrm{mg}$ dose was not developed. In statin-naive patients, recent large trials have demonstrated that statins do appear to lower the rate of CI-AKI and thus become an even stronger mandate for patients with acute or chronic presentations of CAD undergoing percutaneous coronary intervention. Future research should be poised to discover the mechanisms of which statins confer benefits in this scenario with the aim of uncovering additional diagnostic and therapeutic targets.

\section{References}

1 Hsu RK, et al: Temporal changes in incidence of dialysis-requiring AKI. J Am Soc Nephrol 2013;24:37-42.

-2 Nash K, Hafeez A, Hou S: Hospital-acquired renal insufficiency. Am J Kidney Dis 2002;39: 930-936.

-3 Coca SG, Singanamala S, Parikh CR: Chronic kidney disease after acute kidney injury: a systematic review and meta-analysis. Kidney Int 2012;81:442-448.

4 Chertow GM, et al: Preoperative renal risk stratification. Circulation 1997;95:878-884.

5 Thakar CV, et al: Improved survival in acute kidney injury after cardiac surgery. Am J Kidney Dis 2007;50:703-711.

6 Toth PP, Dayspring TD: Drug safety evaluation of rosuvastatin. Expert Opin Drug Saf 2011;10:969-986
7 Teichholz LE: Statin-associated myopathy with normal creatine kinase levels. Ann Intern Med 2003;138:1008, author reply 1008-1009.

8 Graham DJ, et al: Incidence of hospitalized rhabdomyolysis in patients treated with lipidlowering drugs. JAMA 2004;292:2585-2590.

-9 Melli G, Chaudhry V, Cornblath DR: Rhabdomyolysis: an evaluation of 475 hospitalized patients. Medicine (Baltimore) 2005;84:377385.

10 de Meijer AR, et al: Serum creatine kinase as predictor of clinical course in rhabdomyolysis: a 5-year intensive care survey. Intensive Care Med 2003;29:1121-1125.

11 Vidt DG: Statins and proteinuria. Curr Atheroscler Rep 2005;7:351-357.

$12 \mathrm{Wu} \mathrm{Y}$, et al: Effects of rosuvastatin and atorvastatin on renal function: meta-analysis. Circ J 2012;76:1259-1266.
13 Sandhu S, et al: Statins for improving renal outcomes: a meta-analysis. J Am Soc Nephrol 2006;17:2006-2016.

14 Douglas K, O’Malley PG, Jackson JL: Metaanalysis: the effect of statins on albuminuria. Ann Intern Med 2006;145:117-124.

15 Strippoli GF, et al: Effects of statins in patients with chronic kidney disease: meta-analysis and meta-regression of randomised controlled trials. BMJ 2008;336:645-651.

16 Navaneethan SD, et al: HMG CoA reductase inhibitors (statins) for people with chronic kidney disease not requiring dialysis. Cochrane Database Syst Rev 2009:CD007784.

17 Pappy R, et al: Effect of statin therapy on contrast-induced nephropathy after coronary angiography: a meta-analysis. Int J Cardiol 2011; 151:348-353. 
18 Zhang L, et al: Efficacy of statin pretreatment for the prevention of contrast-induced nephropathy: a meta-analysis of randomised controlled trials. Int J Clin Pract 2011;65:624630.

19 Zhang BC, Li WM, Xu YW: High-dose statin pretreatment for the prevention of contrastinduced nephropathy: a meta-analysis. Can J Cardiol 2011;27:851-858.

20 Zhou Y, et al: Short-term, high-dose statins in the prevention of contrast-induced nephropathy: a systematic review and meta-analysis. Clin Nephrol 2011;76:475-483.
21 Li Y, et al: Efficacy of short-term high-dose statin in preventing contrast-induced nephropathy: a meta-analysis of seven randomized controlled trials. PLoS One 2012; 7:e34450.

22 Leoncini M, et al: Early high-dose rosuvastatin for contrast-induced nephropathy prevention in acute coronary syndrome: results from the PRATO-ACS Study (Protective effect of Rosuvastatin and Antiplatelet Therapy On contrast-induced acute kidney injury and myocardial damage in patients with Acute Coronary Syndrome). J Am Coll Cardiol 2014;63:71-79.
23 Han Y, et al: Short-term rosuvastatin therapy for prevention of contrast-induced acute kidney injury in patients with diabetes and chronic kidney disease. J Am Coll Cardiol 2014;63:62-70.

24 Liao JK: Isoprenoids as mediators of the biological effects of statins. J Clin Invest 2002; 110:285-288.

25 Sidaway JE, et al: Inhibitors of 3-hydroxy3-methylglutaryl-CoA reductase reduce receptor-mediated endocytosis in opossum kidney cells. J Am Soc Nephrol 2004;15:22582265. 